\title{
A COVID-19 na Baixada Fluminense: Colapso e apreensão a partir da periferia metropolitana do Rio de Janeiro
}

COVID-19 in Baixada Fluminense: Collapse and seizure from the metropolitan periphery of Rio de Janeiro

COVID-19 en Baixada Fluminense: Colapso y preocupación de la periferia metropolitana de Río de Janeiro

La covid-19 dans la Baixada Fluminense : collapse et appréhension à partir de la périphérie métropolitaine de Rio de Janeiro

\section{Alexandre Fortes, Leandro Dias de Oliveira e Gustavo Mota de Sousa}

\section{OpenEdition}

Journals

\section{Edição electrónica}

URL: http://journals.openedition.org/espacoeconomia/13591

DOI: 10.4000/espacoeconomia. 13591

ISSN: 2317-7837

\section{Editora}

Núcleo de Pesquisa Espaço \& Economia

Refêrencia eletrónica

Alexandre Fortes, Leandro Dias de Oliveira e Gustavo Mota de Sousa, « A COVID-19 na Baixada Fluminense: Colapso e apreensão a partir da periferia metropolitana do Rio de Janeiro », Espaço e Economia [Online], 18 | 2020, posto online no dia 21 abril 2020, consultado o 10 dezembro 2020. URL : http://journals.openedition.org/espacoeconomia/13591 ; DOl : https://doi.org/10.4000/ espacoeconomia.13591

Este documento foi criado de forma automática no dia 10 dezembro 2020.

\section{cc) (†) (ㅇ)}

Espaço e Economia - Revista brasileira de geografia econômica est mise à disposition selon les termes de la licence Creative Commons Attribution - Pas d'Utilisation Commerciale - Partage dans les Mêmes Conditions 4.0 International. 


\title{
A COVID-19 na Baixada Fluminense: Colapso e apreensão a partir da periferia metropolitana do Rio de Janeiro
}

\author{
COVID-19 in Baixada Fluminense: Collapse and seizure from the metropolitan \\ periphery of Rio de Janeiro \\ COVID-19 en Baixada Fluminense: Colapso y preocupación de la periferia \\ metropolitana de Río de Janeiro \\ La covid-19 dans la Baixada Fluminense : collapse et appréhension à partir de la \\ périphérie métropolitaine de Rio de Janeiro
}

Alexandre Fortes, Leandro Dias de Oliveira e Gustavo Mota de Sousa

\section{NOTA DO EDITOR}

Este artigo é fruto dos esforços coletivos realizados no âmbito do Laboratório Aberto do Programa de Pós-Graduação Interdisciplinar em Humanidades Digitais da Universidade Federal Rural do Rio de Janeiro (Open Lab, PPGIHD-UFRRJ, disponível em: https:// www.ppgihd-open-lab.com/), cujo objetivo é agregar conhecimento científico para o enfrentamento da pandemia da COVID-19. Envolvendo a investigação de dinâmicas sociais em escalas variadas através de abordagens combinando análises ou previsões qualitativas e quantitativas, manipulando massas de informação suficientemente grandes ou complexas para exigir sofisticados conceitos, teorias e artefatos computacionais e transitando na interseção entre as diversas disciplinas de Ciências Humanas e de Ciência da Computação, o laboratório tem como escopo modelar cenários, construir mapas digitais, tabular e tratar dados e construir um verdadeiro catálogo de informações disponíveis a todos. Especificamente no que se refere à Baixada Fluminense, o trabalho tem sido realizado em parceria com o Programa de Pós- 
Graduação em Geografia (PPGGEO-UFRRJ), produzindo-se mapas, tabelas e análises diversas, que são disponibilizados de maneira on-line para todo o interessado em informações atualizadas.

\section{Introdução}

1 A análise dos dados ${ }^{1}$ relativos à pandemia de COVID-19 e a construção de cenários estimando os números de infectados e de óbitos têm se revelado tarefas árduas ${ }^{2}$ mesmo para os maiores especialistas das mais consagradas instituições de pesquisa em diversas partes do mundo. Este problema se apresenta de forma particularmente grave na Baixada Fluminense, que padece historicamente de uma profunda carência de dados e indicadores específicos para subsidiar a formulação de políticas para o enfrentamento da sua dramática realidade social.

2 Como pesquisadores vinculados a esse contexto regional, entendemos que é nosso dever colaborar no processamento de informações que possam trazer alguma contribuição para a análise de tendências em relação a esse fenômeno de dimensões inéditas. Mesmo sem formação específica na análise de fenômenos epidemiológicos, utilizamos nossa experiência na análise de fontes variadas de informação para contribuir na complexa tarefa de geração de conhecimentos que possam contribuir para a compreensão da profundidade e complexidade dos desafios decorrentes da presente pandemia, cumprindo também um dever institucional de contribuir cientificamente com o enfrentamento dessa pandemia ${ }^{3}$.

3 Desta maneira, no momento em que escrevemos, as estatísticas globais ${ }^{4}$ registram mais de dois milhões e duzentos mil infectados e mais de cento e quarenta e oito mil mortes provocadas pela COVID-19. No Brasil ${ }^{5}$, ultrapassamos os trinta mil casos e nos aproximamos de 2000 mortos. No estado do Rio de Janeiro, são 3.944 casos e 300 mortos. Na Baixada Fluminense, até o dia 16 de abril, registravam-se 540 casos confirmados e 49 mortes (Ver: mapa 1). Esses números, num primeiro momento, podem parecer pouco expressivos - principalmente numa área onde a vida é comumente desvalorizada pelas autoridades governamentais ${ }^{6}-$, mas constituem apenas a ponta de um iceberg que, ao que tudo indica, atingirá a região de forma desastrosa. 


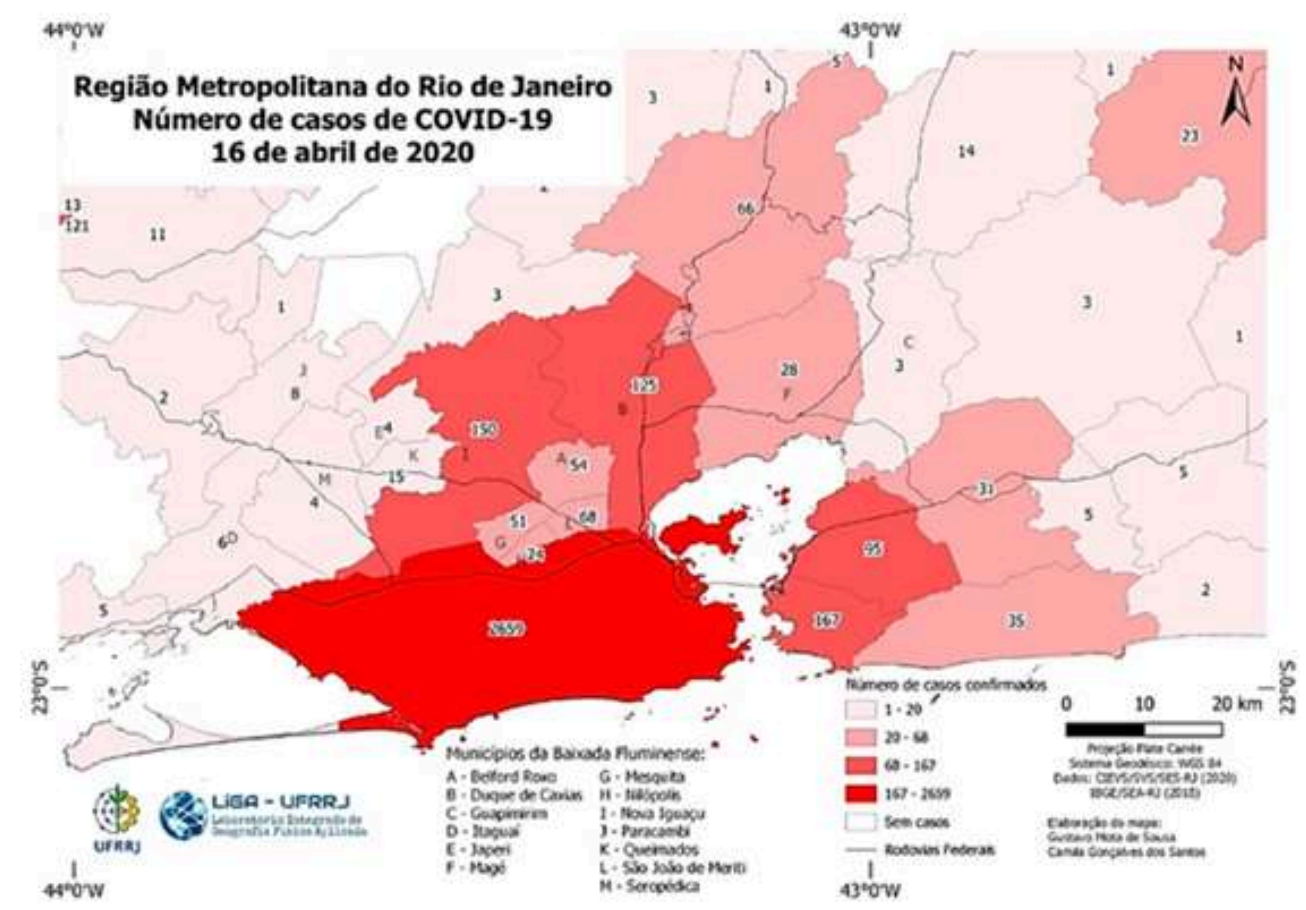

Mapa 1: Número de casos da COVID-19 na Região Metropolitana do Rio de Janeiro.

Fonte: Mapa desenvolvido pelo Laboratório Integrado de Geografia Física Aplicada (LiGA-UFRRJ), disponível em: https://www.ppgihd-open-lab.com/dados-baixada.

4 É importante ressaltar que projeções referentes à evolução da pandemia de COVID-19 em diversas partes do mundo vêm sendo atualizadas diariamente com base na análise de uma série de fatores e no refinamento dos modelos estatísticos utilizados. No Brasil, a fragilidade dos dados, resultante da baixa aplicação de testes e da subnotificação, tem criado uma falsa percepção tanto da dimensão do problema quanto do ponto da curva epidêmica em que nos localizamos no momento. Certos segmentos políticos e sociais chegam a afirmar, na contramão do consenso da comunidade científica, que o pico da epidemia está prestes a ser atingido e que é necessário planejar a retomada das atividades normais.

Considerando as tendências internacionais, é inimaginável que os atuais números mensurados no país representem algo remotamente próximo ao auge da pandemia no Brasil. Na verdade, estima-se que o número real de casos atuais já seja de doze a quinze vezes maior do que o oficialmente registrado 7 . 0 número de mortos pela COVID-19, por sua vez, só será avaliado adequadamente a posteriori, quando for possível comparar os óbitos de cada mês deste ano com as médias históricas do país, já que não está sendo possível testar e/ou processar os exames referentes a as mortes suspeitas. Entretanto, por mais incompletos e imprecisos que sejam os dados disponíveis, eles podem servir como indicadores preciosos de tendências, particularmente quando são desagregados e analisados levando em consideração a expressão territorial das desigualdades sociais.

6 Assim, o objetivo deste artigo é realizar uma reflexão sobre a pandemia no contexto da Baixada Fluminense. Com base em análises realizadas no âmbito do OpenLab do PPGIHD-UFRRJ, são objetivos específicos: [1] entender o contexto da dispersão da pandemia para além da metrópole fluminense; [2] atentar para o impacto da COVID-19 
em uma área historicamente segregada como a Baixada Fluminense e com condições precárias de saúde e moradia para grande parte de sua população.

\section{Para além da capital: os eixos geográficos de dispersão da pandemia}

7 O estado do Rio de Janeiro apresenta uma condição histórica cuja análise é fundamental em tempos de pandemia. Trata-se de um estado cuja capital - a cidade do Rio de Janeiro, durante muito tempo uma cidade autônoma e capital federal ${ }^{8}$ - concentra historicamente os investimentos econômicos, produtivos, logísticos e sociais e apresenta integração ainda restrita com interior ${ }^{9}$. Mesmo com o crescimento produtivo-industrial de certas áreas do interior, como os novos empreendimentos localizados na região do Médio Vale do Paraíba Fluminense (OLIVEIRA, 2008) ou da consolidação da economia do petróleo no Norte Fluminense (PESSANHA, 2017), a cidade do Rio de Janeiro permanece, por meio de suas periferias imediatas e com os eixos de contato logístico, como catalizador metropolitano, especialmente no que se refere ao atendimento das necessidades de educação e saúde.

A região metropolitana, da mesma maneira, também pode ser dividida geograficamente: além da capital, há uma nítida dissociação entre as cidades do Leste Fluminense - sendo a antiga capital do estado do Rio de Janeiro, Niterói, a mais importante - e Baixada Fluminense, com destaque para as cidades de Nova Iguaçu e Duque de Caxias. A própria Baixada Fluminense também apresenta diferenças, especialmente entre a baixada histórica, de origem iguaçuana e com urbanização mais densificada - que passa por uma reestruturação urbano-econômica (ROCHA, 2015), e a porção do extremo oeste metropolitano formado pelas cidades de Seropédica, Itaguaí, Paracambi, Japeri e Queimados ${ }^{10}$ (OLIVEIRA, 2014), além de Magé e Guapimirim, que compõem uma transição Baixada-Leste Metropolitano (OLIVEIRA, 2020) (Ver: mapa 2). o extremo oeste metropolitano apresenta características menos "urbanas" e um processo de reestruturação apoiado nos investimentos significativos que recebeu nos últimos anos por conta de novos empreendimentos logísticos e industriais. Assim, se por um lado, emergiu uma economia de "borda metropolitana" - a construção do Arco Rodoviário Metropolitano Fluminense é a evidência mais importante desse processo de construção de uma economia para além da capital e cujos aportes ocorrem especialmente nas franjas metropolitanas -, o acesso a serviços permanece centrado na capital. Tais diferenças histórico-geográficas implicam no adensamento populacional das cidades da "Baixada Iguaçuana", tornando-as mais suscetíveis ao aumento dos casos $^{11}$; todavia, tais cidades de urbanização mais consolidada possuem - mesmo com precariedade em termos de leitos hospitalares e vagas em UTI - mais equipamentos médicos e leitos disponíveis, conforme nos apresenta André Rocha $(2020)^{12}$. 


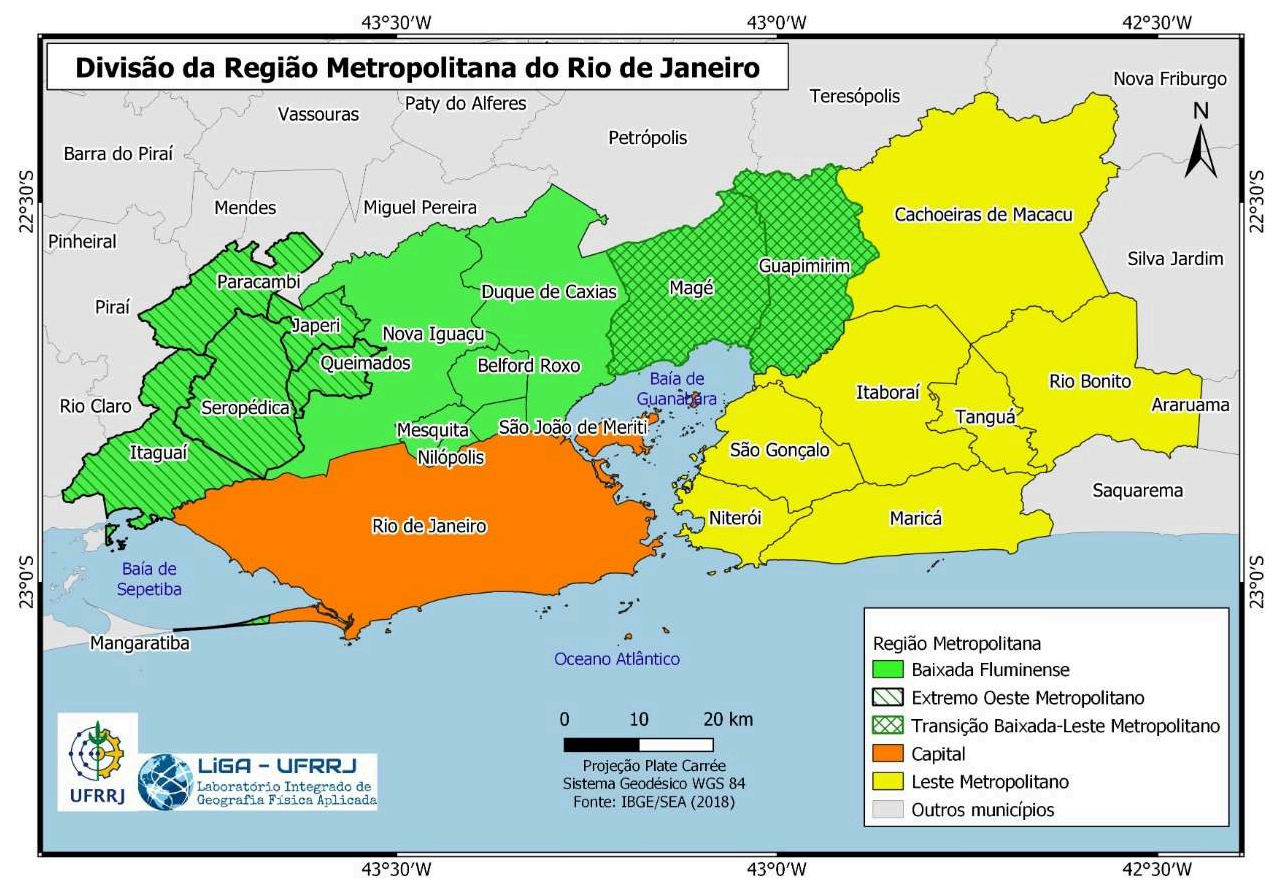

Mapa 2: A Região Metropolitana do Rio de Janeiro: capital, Leste Metropolitano e Baixada Fluminense, subdividida, além da Baixada "Histórica-Iguaçuana", em "Transição Baixada-Leste Metropolitano" e Extremo Oeste Metropolitano.

Organização e Elaboração: Laboratório Integrado de Geografia Física Aplicada (LiGA-UFRRJ) e Laboratório de Geografia Econômica e Política (LAGEP-UFRRJ) [2020].

Assim, os limites da região metropolitana do Rio de Janeiro se tornam fluídos, fazendo com que suas fronteiras se tornem verdadeiro propagadores do desenvolvimento econômico. Nesta mesma seara, as rodovias principais do estado se tornam eixos geográficos de expansão metropolitana, conectando-a com a Região do Médio Vale Paraíba Fluminense, com destaque econômico para a cidade de Volta Redonda; a Região dos Lagos (ou Baixadas Litorâneas), onde se sobressai Cabo Frio; e mesmo o Norte Fluminense, com destaque para a cidade de Campos dos Goytacazes. Expressando características metropolitanas a partir da influência e força da capital, na realidade existem diferentes graus de integração a metrópole por todo o estado do Rio de Janeiro.

Neste sentido, a dispersão do coronavírus na realidade não deixa de ser uma lição de geografia econômica da metrópole, afinal, os mesmos sentidos de metropolização em quaisquer atividades econômicas tradicionais também parecem reverberar nos processos de espraiamento geográfico de contaminação da COVID-19 no estado. 0 mapa a seguir (mapa 3), produzido pelo Laboratório Integrado de Geografia Física Aplicada (LiGA) da UFRRJ, apresenta os números de contaminação do estado do Rio de Janeiro expostos sobre a malha rodoviária federal, portanto, destacando as principais estradas federais no estado do Rio de Janeiro. 


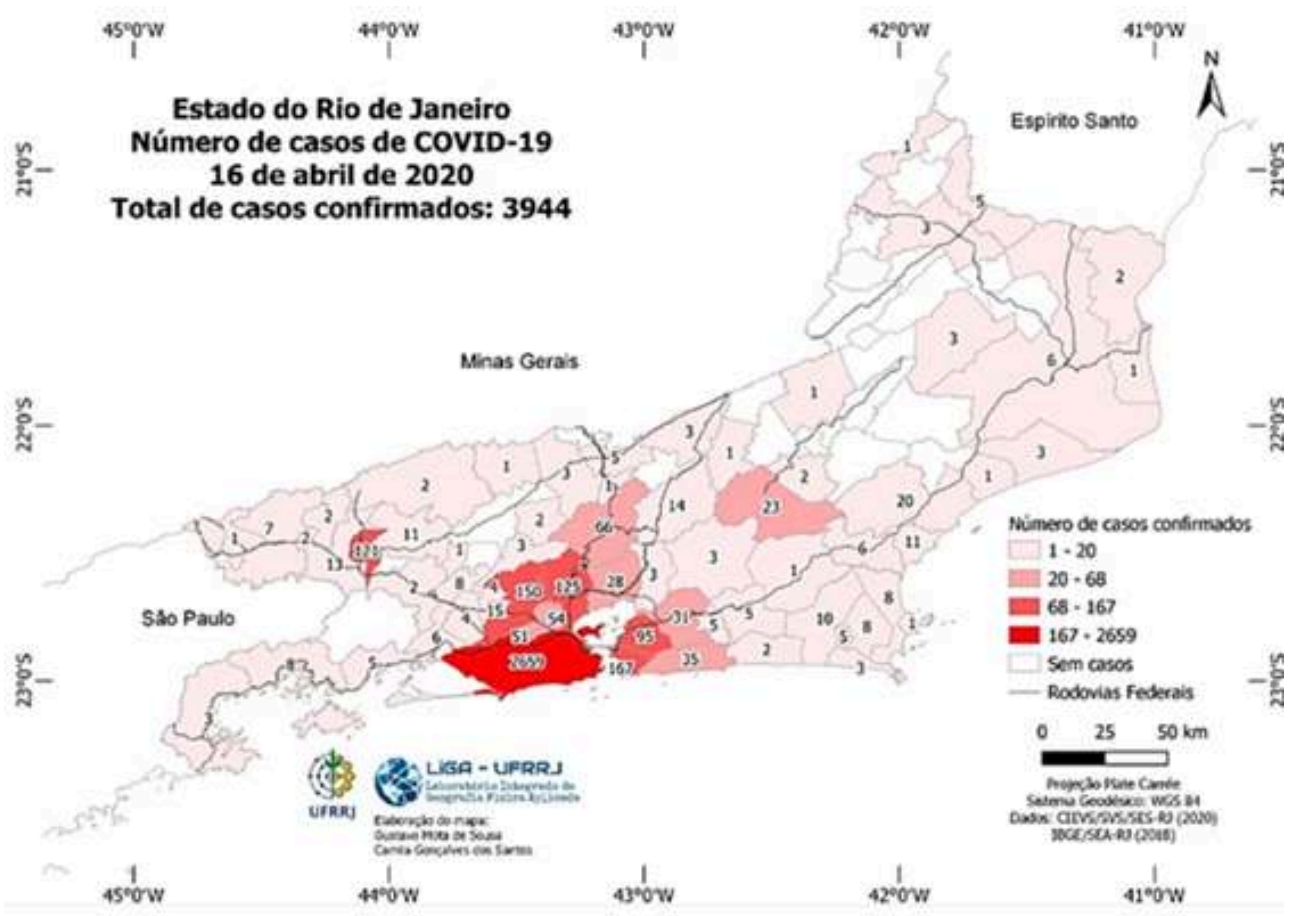

Mapa 3: Número de contaminados e eixos rodoviários e de dispersão do coronavírus, em 16 de abril de 2020.

ORgANIZAÇÃo E ELABORAÇÃO: LABORATÓRIO INTEgRAdo dE GEOgRAfiA FísICA APLICADA (LIGAUFRRJ), 2020.

De maneira geral, os eixos rodoviários - federais, mas também os estaduais - revelam as vias de propagação da COVID-19 no estado do Rio de Janeiro (OLIVEIRA, 2020) ${ }^{13}$. As cidades que polarizam as atividades regionais - que também são aquelas com maior população e centros urbanos mais robustos - também devem apresentar maiores casos de contaminação e de óbitos no interior do estado. Volta Redonda (com 121 casos oficiais, sempre destacando as expressivas subnotificações), Cabo Frio (8), Campos dos Goytacazes (6), Macaé (20), Angra dos Reis (8), entre outras, se tornam progressivamente polos propagadores do coronavírus.

O caso de Volta Redonda é emblemático: além de ser uma cidade de classe média substantiva, com o centro comercial regionalmente mais importante, de abarcar muitos trabalhadores da cidades vizinhas - especialmente pelo papel histórico da companhia Siderúrgica Nacional (MOREIRA, 2003) -, e ainda apresentar número bastante elevado de pessoas com problemas respiratórios, devido à presença da própria Usina Presidente Vargas (nome da planta industrial da CSN na cidade) ${ }^{14}$, a cidade se situa justamente no eixo Rio-São Paulo, no caminho da Rodovia Presidente Dutra (BR-116)

O contato metropolitano entre as mais importantes cidades do país, Rio de Janeiro e São Paulo, é algo de maior relevo desde que Getúlio Vargas, então Presidente da República, decidiu pela construção da CSN no então oitavo distrito de Barra Mansa, Santo Antônio de Volta Redonda, ainda na década de 1940 (OLIVEIRA, 2006). A Rodovia Presidente Dutra, construída apenas em 1951, se consolidou como a mais importante economicamente da Brasil e épor onde circula grande parte das mercadorias produzidas entre as duas metrópoles nacionais. Essa grande configuração metropolitana constitui uma potente economia macrorregional, cujas densidades 
técnicas, produtivas, logísticas e estratégicas formam, nas palavras Sandra Lencioni (2014), uma verdadeira "nebulosa metropolitana", que permanece em expansão geográfica. Volta Redonda, em meio a este cenário, continua até o presente momento atrás somente da capital e de Niterói em número de contaminados.

Com tamanha centralidade na capital, as preocupações acerca da disponibilidade de equipamentos médicos, da logística hospitalar, da estrutura de acolhimento e mesmo da capacidade de triagem e atendimento nos casos mais graves da COVID-19 são muito significativas, tanto nas periferias imediatas da metrópole quanto no interior. É tradição no estado do Rio de Janeiro procurar a capital para resolução que casos mais graves de saúde; portanto, é justamente no momento em que se necessita de maior espraiamento dos serviços públicos médico-hospitalares é que se revelam as graves diferenças e a imensa segregação entre capital e interior, e mesmo entre capital e seu entorno imediato.

No caso da Região Metropolitana do Rio de Janeiro, os painéis de dados atualizados pelas secretarias de saúde do estado e do município do Rio de Janeiro oferecem informações sobre a distribuição dos casos e óbitos por municípios, bairros e regiões administrativas.

Os casos confirmados na capital até esta semana ainda se concentram na Área de Planejamento 2, que congrega a Zona Sul e Grande Tijuca, particularmente na AP 2.1, composta pelos bairros oceânicos como Leblon, Ipanema e Copacabana. Em segundo lugar, vem a AP 4, que inclui Barra e Jacarepaguá. A disparidade entre o índice de casos confirmados por 100 mil habitantes nas várias APs é impressionante, variando de 85,61 na AP 2 para apenas 12,14 na populosa AP 5 (Campo Grande, Bangu, Santa Cruz, etc.), cujo perfil socioeconômico se assemelha em muito ao dos municípios da Baixada.

Tabela 1: Número de contaminados nas Áreas de Planejamento da cidade do Rio de Janeiro

\begin{tabular}{|l|r|r|r|}
\hline \multicolumn{1}{|c|}{ 13042020 } & Populasio & Casos & $\begin{array}{l}\text { Casos por } \\
\text { 100 milhab. }\end{array}$ \\
\hline AP 1 (Centro) & 297976 & 93 & 31,21 \\
\hline AP 2 (Zona Sul) & 1009170 & 864 & 85,61 \\
\hline AP 3 (Zona Norte) & 2398572 & 520 & 21,68 \\
\hline AP 4 (Barra) & 909955 & 380 & 41,76 \\
\hline AP 5 (Campo Grande) & 1704773 & 207 & 12,14 \\
\hline Total & $\mathbf{6 3 2 0 4 4 6}$ & $\mathbf{2 0 6 4}$ & $\mathbf{3 2 , 6 6}$ \\
\hline
\end{tabular}

Organização: Prof. Dr. Alexandre Fortes, com dados da Secretaria de Saúde da cidade do Rio de Janeiro.

17 Há duas explicações possíveis para a persistência dessas disparidades: 1) os índices de subnotificação são maiores nos bairros mais pobres; 2) o isolamento mais efetivo nos bairros de elite está retardando o avanço da pandemia na periferia. Ou seja, é possível que a redução do contato da classe média alta que concentra o maior percentual de infectados com os trabalhadores dos setores de comércio e serviços que residem nos bairros e municípios periféricos esteja reduzindo a velocidade de difusão do vírus no conjunto da Região Metropolitana. Entretanto, qualquer que seja a proporção na combinação desses dois fatores (subnotificação e isolamento social), a multiplicação exponencial do número de contaminados das áreas com condições de vida mais precárias da Região Metropolitana é apenas uma questão de tempo. 

COVID-19. Ampliar a capacidade de testagem e atendimento e diluir o número de casos graves num período mais longo para evitar o colapso do sistema de saúde são, no momento, os principais objetivos dos gestores do sistema de saúde em todo o mundo. Resta saber o quanto a Baixada Fluminense terá condições de aproveitar esse tempo (FORTES, 2020).

\section{Baixada Fluminense: sinais de um tsunami pandêmico}

19 A Baixada Fluminense pode ser atingida por um verdadeiro tsunami de contaminados com a expansão metropolitana da pandemia da COVID-19. Essa dura previsão se deve a vários fatores: 1) A dinâmica da pandemia tem levado, em todo mundo, a uma dificuldade em acompanhar o número de infectados, e até mesmo de óbitos; 2) o Brasil deve presenciar, nas próximas semanas, o crescimento exponencial do número de infectados; 3) A COVID-19 se difundiu no Rio de Janeiro inicialmente entre segmentos com condições mais favoráveis, e tudo indica que ganhará uma dinâmica muito mais agressiva nas periferias urbanas; 4) A alta densidade demográfica, a precariedade das condições de vida e das unidades de saúde ${ }^{15}$, assim como a influência de lideranças negacionistas tendem a agravar o impacto da doença na região (FORTES, OLIVEIRA, 2020). Examinemos brevemente cada um desses aspectos.

A rápida difusão global da COVID-19 pegou de surpresa mesmo os melhores sistemas de saúde do mundo. Na grande maioria dos países, a escassez de testes seguros disponíveis para aplicação em larga escala gerou estatísticas falhas e incompletas. Matéria recente da revista The Economist aponta que tanto no norte da Itália quanto no centro da Espanha o número de óbitos acima da média histórica durante os momentos de pico da epidemia foi duas a três vezes superior ao de mortes oficialmente associadas ao novo coronavírus ${ }^{16}$. O New York Times prevê que o número real de mortos pela pandemia nos EUA não será conhecido até $2021^{17}$.

No Brasil, um grupo de especialistas de diversas instituições avalia que o número real de infectados deveria estar na casa dos $82 \mathrm{mil}^{18}$ no dia 07 de abril, quando o registro oficial contabilizava cerca de 12 mil. Ou seja, o número real deve ser cerca de sete vezes maior do que indicam as estatísticas ${ }^{19}$. Em São Paulo, o número de enterros realizados nos cemitérios já cresceu numa proporção muito superior à dos casos confirmados de COVID-1920.

Esses números, por um lado, podem gerar uma percepção de uma letalidade muito superior à real, já que apenas pacientes graves e mortos são testados. De outro lado, porém, eles indicam que o número de infectados sem sintomas ou com sintomas leves em circulação é muito mais alto do que se supõe, o que torna provável uma explosão de casos em breve, como indica a figura (Figura 1) abaixo, gerada pelo Painel Coronavírus Brasil, no dia 17 de abril ${ }^{21}$. 


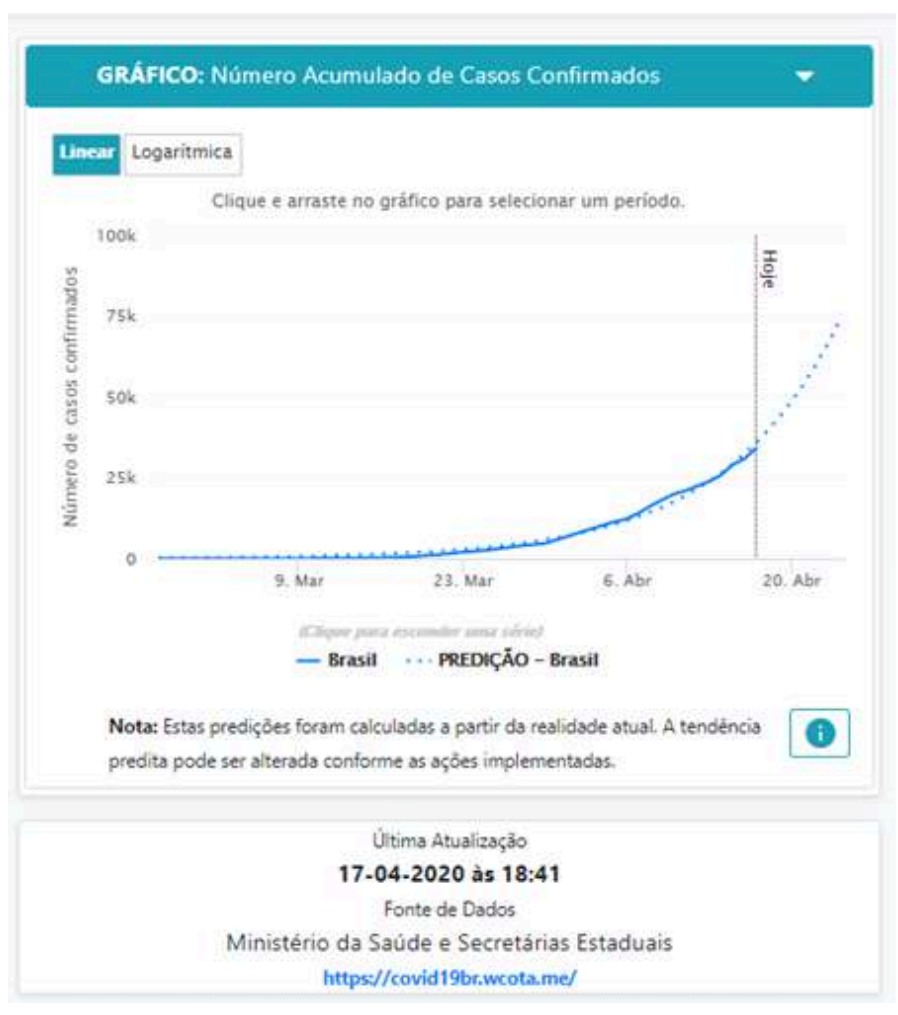

Figura 1: Número acumulado e predição de casos confirmados no Brasil

Fonte: http://painel.covid19br.org/. Acesso em: 17 de abril de 2020.

O conjunto de fatores comentados acima fundamenta a hipótese de que o Brasil deve se defrontar em breve com um salto que hoje parece quase inimaginável na dimensão do problema. Instituições especializadas trabalham com projeções que estabelecem como cenário mais otimista um número total de 200 mil mortos no país, chegando, num cenário pessimista, a 2 milhões de óbitos ${ }^{22}$.

4 A instalação da pandemia como um fenômeno de massas na Baixada e nas demais áreas periféricas da Região Metropolitana do Rio de Janeiro, entretanto, vem sendo em certa medida retardada pelas características da difusão da doença no estado. Tudo indica que o principal vetor da introdução do vírus no Rio de Janeiro foi a elite globalizada (pessoas que viajam ao exterior ou tem contato com quem viaja), à qual se somaram, possivelmente, grupos de turistas em visita à capital. Isso explica por que os casos se concentram inicialmente em locais turísticos e de renda elevada.

5 Essa característica socioeconômica da primeira fase da instalação da pandemia no estado pode ser percebida até mesmo na disparidade dos dados sobre os diferentes bairros da capital. No dia 07 de abril, a Barra, com 650 mil habitantes, tinha 102 casos confirmados. Já Campo Grande e Bangu, que somados, tem quase a mesma população, registravam apenas 10 casos. Leblon, Copacabana, Ipanema, Botafogo, Lagoa, Tijuca, Centro, Flamengo e São Conrado, vários deles com populações relativamente pequenas, completavam a lista dos dez bairros que mais concentravam casos e óbitos.

Esses bairros de classe média alta certamente oferecem melhores condições para isolamento social e maior acesso à rede de saúde. A notificação tende a ser mais alta, mas a curva vai achatar mais rapidamente e o tratamento dos casos graves será melhor, até porque estão chegando mais cedo a bons hospitais e às UTIs. Nas próximas semanas, 
ao que tudo indica, a epidemia vai explodir nas favelas, assim como nas partes mais pobres das Zonas Norte e Oeste e na Baixada Fluminense, com taxas de transmissão muito mais aceleradas e letalidade muito mais alta.

A dinâmica da pandemia em contextos de extrema desigualdade social e precariedade de condições de vida ainda não é plenamente conhecida, mas tudo indica que será avassaladora. Nos Estados Unidos, os dados já demonstram que a doença atinge de forma desproporcionalmente alta e de maneira muito mais agressiva a população afroamericana, cuja vulnerabilidade no que diz respeito a saúde, ocupação, renda, habitação e saneamento predispõe ao contágio e ao agravamento do quadro ${ }^{23}$. Conforme argumenta um colunista do New York Times, nesses cenários não se pode ignorar que a possibilidade de desfrutar de um efetivo distanciamento social é um privilégio ${ }^{24}$. O portal UOL, em matéria recente ${ }^{25}$, alerta para a combinação explosiva entre a persistência ou ressurgência de doenças negligenciadas como a Tuberculose e a COVID-19 em favelas cariocas. A realidade da Baixada certamente não é diferente.

Trata-se de uma região onde a mortalidade infantil média nos treze municípios é de $15 \%$, acima da média nacional de $12,4 \%$ e do índice da capital, de 11,2\%. Em Nilópolis, ele chega a $21 \%$. Tais dados revelam a precariedade das condições de habitação, acesso a água corrente e saneamento, que devem ter forte influência na dificuldade de conter a circulação do coronavírus. Da mesma maneira, os 95 casos de tuberculose por 100.000 habitantes da Baixada no ano de 2018 representam 2,5 vezes a média nacional (FORTES, 2020).

29 Entretanto, a população e muitas das lideranças sociais, políticas ${ }^{26}$ e religiosas locais parecem não compreender a gravidade da situação. Apesar da estrutura de atendimento em saúde da região estar completamente desaparelhadas para enfrentar um tsunami como o que se aproxima, os relatos indicam a persistência de atividades frontalmente contrárias a qualquer diretriz visando reduzir o potencial de multiplicação do número de infectados ${ }^{27}$. Há que se temer, por outro lado, que uma vez instaurado um cenário de grande número de mortos e infectados, o estigma que historicamente já torna a população da Baixada Fluminense vítima de preconceito seja reforçado, e as políticas de contenção adotadas tenham forte conteúdo repressivo e de desrespeito aos direitos humanos.

30 É fundamental que todos atores sociais com melhor acesso a informações científicas consistentes e confiáveis contribuam para alertar a população e as autoridades sobre os riscos que se abatem sobre o Brasil em geral e sobre a Baixada Fluminense em particular.

\section{Considerações Finais}

31 Em linhas gerais, podemos assim sintetizar as reflexões aqui empreendidas: [1] mesmo sabendo que os dados oficiais atuais são subestimados, está claro que, até o momento, a COVID-19 na Região Metropolitana do Rio de Janeiro se concentra em áreas de melhor renda e maior acesso a serviços de saúde; [2] o isolamento social razoavelmente efetivo da classe média carioca pode estar contribuindo para proporcionar um tempo precioso aos gestores públicos na preparação para enfrentar o pico da pandemia; [3] os índices relativos a condições sanitárias na Baixada Fluminense (e, provavelmente, na parte do município do Rio de Janeiro ainda pouco atingida pelo contágio) apontam para um 
cenário de imensa gravidade à medida que se estabeleça a dinâmica de "expansão acelerada" nesses locais.

Mike Davis (2006 [2005], p. 198), em sua obra "O monstro bate a nossa porta: a ameaça global da gripe aviária" faz uma importante indagação : como reagiriam as cidades quase indefesas do terceiro mundo a uma pandemia? Comungamos desta preocupação, afinal, a Baixada Fluminense se revela um espaço onde a pandemia de coronavírus pode se revelar profundamente mais agressiva. Com uma população adensada, em grande parte com precárias condições de vida, com instalações e equipamentos médicos insuficientes em seu território e grande dificuldade de promover distanciamento social - seja pelas necessidades de constituir renda, seja pelas próprias limitações das habitações -, a Baixada Fluminense pode se tornar um triste laboratório capaz de responder as preocupações de Mike Davis.

Por sua vez, o geógrafo brasileiro Ruy Moreira (1987), em "O Discurso do Avesso: para a crítica da geografia que se ensina" define como "homem estatístico" o exato momento que o indivíduo se torna número na reflexão geográfica. Com a primazia da estatística, perde-se o sentido socioemocional da vida. Convictos de que o número de contaminados pela COVID-19 é superior ao divulgado pelas instâncias governamentais, entendemos que a subnotificação de casos corresponde hoje ao maior desafio para a compreensão das curvas de contágio. Com um número tão pequeno de testes, somos incapazes de saber exatamente o estágio de contaminação, o número de pessoas recuperadas e mesmo o quantitativo de óbitos oriundos da pandemia. Entretanto, acima do interesse de traçar um perfil correto do momento da pandemia, reforçamos que toda a vida humana é muito importante. $O$ valor de uma vida é imensurável e o impacto de um óbito para seus amigos e familiares não pode ser visto com um número frio.

Devemos, portanto, enfrentar os atuais discursos de desvalorização da vida por parte de lideranças políticas nacionais. A relativização para com o número anunciado de óbitos pelas estruturas governamentais e a supremacia das preocupações econômicas sobre as próprias estratégias de contenção do vírus reforçam o desprezo pela vida especialmente dos mais pobres, negros e segregados territorialmente - de certas autoridades. Assim como mundialmente a COVID-19 tem sido frequentemente atribuída às práticas culturais "impuras" dos chineses pobres de Wuhan (COLETIVO CHUANG, 2020), em tempos de individualismo, concorrência mercadológica, preconceitos exacerbados e intolerância política, há a sincera preocupação que se culpabilize, na Baixada Fluminense, as próprias vítimas.

\section{BIBLIOGRAFIA}

ALVES, José Cláudio de Souza. Dos Barões ao Extermínio: Uma História da Violência na Baixada Fluminense. Duque de Caxias: APPH-CLIO, 2003. 
ALVES, José Cláudio de Souza. Coronavírus e milícia: a morte favorece a quem? Contrapoder, 2020. Disponível em: https://contrapoder.net/colunas/coronavirus-e-milicia-a-morte-favorecea-quem/. Acesso em: 17 de abril de 2020.

BOLETIM de Análise da Conjuntura, ano 05, nº 45, Fundação Perseu Abramo, março/abril de 2020. Consultar: https://fpabramo.org.br/wp-content/uploads/2020/04/Boletim-mensal-analiseconjuntura-45-territorial.pdf. Acesso em: 20 de abril de 2020.

COLETIVO CHUANG. Contágio Social: coronavírus e a luta de classes microbiológica na China. São Paulo : Veneta, 2020.

DAVIDOVICH, Fani Raquel. Estado do Rio de Janeiro: singularidades de um contexto territorial. Território, Rio de Janeiro: LAGET/UFRJ, nº9, p.9-24, jul-dez.2000. Disponível em: http:// www.laget.eco.br/pdf/09_2_davidovich.pdf. Acesso em: 17 de abril de 2019.

DAVIS, Mike. 0 monstro bate a nossa porta: a ameaça global da gripe aviária. Rio de Janeiro : Record, 2006 [2005].

FARIAS, Heitor Soares de. A Dispersão da Covid-19 na Baixada Fluminense: um paralelo entre Duque de Caxias e Nova Iguaçu. OpenLab / PPGIHD-UFRRJ, 2020. Disponível em: https:// www.ppgihd-open-lab.com/post/a-dispers\%C3\%A3o-da-covid-19-na-baixada-fluminense-umparalelo-entre-duque-de-caxias-e-nova-igua\%C3\%A7u. Acesso em 20 de abril de 2020.

FORTES, Alexandre. Dinâmica socioespacial da COVID-19 na cidade do Rio de Janeiro e na Baixada Fluminense. OpenLab / PPGIHD-UFRRJ, 2020. Disponível em: https://www.ppgihd-open-lab.com/ post/din\%C3\%A2mica-socioespacial-da-covid-19-na-cidade-do-rio-de-janeiro-e-na-baixadafluminense. Acesso em: 20 de abril de 2019.

FORTES, Alexandre; OLIVEIRA, Leandro Dias de. COVID-19 na Baixada Fluminense: 0 tsunami se aproxima. OpenLab / PPGIHD-UFRRJ, 2020. Disponível em: https://www.ppgihd-open-lab.com/ post/covid-19-na-baixada-fluminense-o-tsunami-se-aproxima. Acesso em: 20 de abril de 2019.

LENCIONI, Sandra. Urbanização difusa e a constituição de megarregiões: O caso de São Paulo-Rio

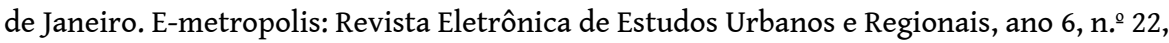
setembro de 2015. Disponível em: http://www.emetropolis.net/index.php? option=com_edicoes\&task=artigos\&id=132\&lang=pt. Acesso em: 17 de abril de 2019.

LESSA, Carlos. O Rio de todos os brasis: uma reflexão em busca de autoestima. Rio de Janeiro/São Paulo: Editora Record, 2000.

MOREIRA, Ruy (org.). A reestruturação Industrial e Espacial do Estado do Rio de Janeiro. Niterói: GERET/NEGT/GECEL, 2003.

NESIMI, Mariana dos Santos; BOTELHO, Maurilio Lima. Vida e morte na cidade: Segregação e exclusão na era da pandemia. OpenLab / PPGIHD-UFRRJ, 2020. Disponível em: https:// www.ppgihd-open-lab.com/post/vida-e-morte-na-cidade-segrega\%C3\%A7\%C3\%A3o-eexclus\%C3\%A3o-na-era-da-pandemia. Acesso em: 20 de abril de 2020.

MOREIRA, Ruy. O Discurso do Avesso: para a crítica da geografia que se ensina. Rio de Janeiro: Dois Pontos, 1987.

OLIVEIRA, Floriano José Godinho. Reestruturação produtiva, território e poder no Rio de Janeiro. Editora Garamond-Faperj, Rio de Janeiro, 2008.

OLIVEIRA, Leandro Dias de. A Construção do Desenvolvimento Sustentável na Cidade de Volta Redonda: Um Estudo sobre Reestruturação do Território e Ideologia, 2006. Dissertação (Mestrado em Geografia). Departamento de Geografia do Instituto de Geociências, Universidade do Estado do Rio de Janeiro, Rio de Janeiro, 2006. 
OLIVEIRA, Leandro Dias de. A emersão da região logístico-industrial do Extremo OesteMetropolitano fluminense: reflexões sobre o processo contemporâneo de reestruturação territorial-produtiva". In: Espaço e Economia: Revista Brasileira de Geografia Econômica, ano IV, n. 7, julho-dezembro de 2015. Disponível em: http://journals.openedition.org/espacoeconomia/ 1814. Acesso em: 17 de abril de 2019.

OLIVEIRA, Leandro Dias de. Para além da capital: os eixos geográficos de dispersão da pandemia no estado do Rio de Janeiro. OpenLab / PPGIHD-UFRRJ, 2020. Disponível em: https:// www.ppgihd-open-lab.com/post/para-al\%C3\%A9m-da-capital. Acesso em: 20 de abril de 2019.

PESSANHA, Roberto Moraes. A relação transescalar e multidimensional "Petróleo-Porto" como produtora de novas territorialidades. Tese (Doutorado em Políticas Públicas e Formação Humana), Centro de Educação e Humanidades, Universidade do Estado do Rio de Janeiro, 2017. Disponível em: http://ppfh.com.br/wp-content/uploads/2018/05/Tese-NORMALIZADA-site.pdf. Acesso em: 17 de abril de 2019.

RIBEIRO, Miguel Angelo. Estado do Rio de Janeiro: das capitanias hereditárias à uma nova divisão regional. Geo UERJ, Rio de Janeiro, n. 31, p. 249-279, 2017. Disponível em: file://C:/Users/Prof. \%20Leandro\%20Dias/Downloads/32064-107038-1-PB.pdf. Acesso em: 17 de abril de 2019.

ROCHA, André Santos da. Os efeitos da reestruturação econômica metropolitana na Baixada Fluminense: Apontamentos sobre o "novo" mercado imobiliário da região. Espaço e Economia: Revista Brasileira de Geografia Econômica, ano III, n. 6, janeiro-junho de 2015. Disponível em: http://journals.openedition.org/espacoeconomia/1677. Acesso em: 17 de abril de 2019.

ROCHA, André Santos da. "Nada é tão ruim que não possa piorar": A COVID-19 e as políticas de saúde na Baixada. OpenLab / PPGIHD-UFRRJ, 2020. Disponível em: https://www.ppgihd-openlab.com/post/nada-\%C3\%A9-t\%C3\%A3o-ruim-que-n\%C3\%A3o-possa-piorar. Acesso em: 17 de abril de 2019.

ROSSI, Fabio. Volta Redonda: Números da pandemia de COVID-19. OpenLab / PPGIHD-UFRRJ, 2020. Disponível em: https://www.ppgihd-open-lab.com/post/volta-redonda-n\%C3\%BAmerosda-pandemia-de-covid-19. Acesso em 17 de abril de 2020.

Páginas eletrônicas consultadas :

http://painel.covid19br.org.

http://portal.ufrrj.br.

https://contrapoder.net.

https://covid.saude.gov.br.

https://estadao.com.br.

https://fpabramo.org.br.

https://noticias.uol.com.br.

https://oglobo.globo.com.

https://portal.fiocruz.br

https://projetocolabora.com.br.

https://www.abrasco.org.br.

https://www.economist.com.

https://www.newscientist.com. 
https://www.ppgihd-open-lab.com.

https://www.nytimes.com.

https://www.vox.com.

https://www.worldometers.info/coronavirus.

\section{NOTAS}

1. Consultar: https://www.vox.com/science-and-health/2020/4/2/21197617/coronaviruspandemic-covid-19-death-rate-transmission-risk-factors-lockdowns-social-distancing. Acesso em 17 de abril de 2020.

2. Consultar: https://www.newscientist.com/article/2239497-why-we-still-dont-know-what-thedeath-rate-is-for-covid-19/. Acesso em 17 de abril de 2020.

3. Torna-se oportuno destacar que a UFRRJ, além de manter as atividades de pesquisa e extensão de forma remota numa comunidade que envolve milhares de estudantes, tem realizado ações que fomentem a ação solidária institucional. Como exemplos: [1] entre 23 de março e 3 de abril, mais de 1.200 litros de álcool $70^{\circ}$ produzidos pelo Instituto de Química (IQ) atenderam para além da comunidade externa, contemplando unidades de saúde e entidades localizadas em municípios da Baixada Fluminense, que já receberam mais de 870 litros do total; [2] o Programa de Fortalecimento da Agricultura Familiar da UFRRJ implementou, em parceria com a Emater-Rio, um sistema de compras de cestas de alimentos da agricultura familiar da região da Baixada e Centro-Sul Fluminense, garantindo a comercialização dos produtos, geração de renda para as famílias e fazer com que os alimentos cheguem até os consumidores. Para além das ações institucionais, uma infinidade de ações solidárias realizadas por professores e demais servidores, alunos de diferentes modalidades e cursos e outros membros da comunidade acadêmica e local, em grande parte de maneira discreta e sem grande alarde, tem proporcionado alimentos, itens básicos de higiene e outros artigos que permitem levar conforto e esperança aos mais necessitados. Consultar: http://portal.ufrrj.br/rural-distribui-mais-de-1-200-litros-de-alcool-70para-comunidades-interna-e-externa/ e http://www.ni.ufrrj.br/ufrrj-implanta-sistema-paracompra-de-cestas-de-produtos-da-agricultura-familiar/. Acesso em: 17 de abril de 2020.

4. Consultar: https://www.worldometers.info/coronavirus/\#countries. Acesso em: 17 de abril de 2020.

5. Consultar: https://covid.saude.gov.br/. Acesso em: 17 de abril de 2020.

6. Ver: ALVES, José Cláudio de Souza. Dos Barões ao Extermínio: Uma História da Violência na Baixada Fluminense. Duque de Caxias: APPH-CLIO, 2003. Do mesmo autor, de longa trajetória nos estudos sobre violência e poder na Baixada Fluminense, consultar também: "Coronavírus e milícia: a morte favorece a quem?", disponível em: https://contrapoder.net/colunas/ coronavirus-e-milicia-a-morte-favorece-a-quem/. Acesso em: 17 de abril de 2020.

7. Consultar: https://noticias.uol.com.br/saude/ultimas-noticias/redacao/2020/04/14/ pesquisas-subnotificacao-casos-confirmados-brasil.htm. Acesso em: 15 de abril de 2020.

8. Após a construção de Brasília e consequente transferência da capital federal na década de 1960, a cidade do Rio de Janeiro transformada numa cidade-estado com o nome de Guanabara. Somente no ano de 1975 ocorreu a fusão com o antigo estado do Rio de Janeiro, tornando a cidade capital estadual. Consultar, entre outros: LESSA, Carlos. O Rio de todos os brasis: uma reflexão em busca de autoestima. Rio de Janeiro/São Paulo: Editora Record, 2000.

9. A fusão, conforme realizada, não impediu que se consolidasse a ausência de integração e identidade entre a capital e o interior (DAVIDOVICH, 2000; RIBEIRO, 2017). 
10. Japeri e Queimados foram emancipadas de Nova Iguaçu, mas apresentam características urbano-econômicas que as aproximam mais do Extremo Oeste Metropolitano; assim, podem ser entendidas como transição entre a "Baixada Histórica" e o "Extremo Oeste" da região metropolitana.

11. Consultar: Boletim de Análise da Conjuntura da Fundação Perseu Abramo, ano 05, oㅡ 45, março/abril 2020. Consultar: https://fpabramo.org.br/wp-content/uploads/2020/04/Boletimmensal-analise-conjuntura-45-territorial.pdf. Acesso em 20 de abril de 2020. Da mesma maneira, consultar a contribuição de Heitor Soares de Farias, "A Dispersão da Covid-19 na Baixada Fluminense: um paralelo entre Duque de Caxias e Nova Iguaçu", disponível em: https:// www.ppgihd-open-lab.com/post/a-dispers\%C3\%A3o-da-covid-19-na-baixada-fluminense-umparalelo-entre-duque-de-caxias-e-nova-igua\%C3\%A7u. Acesso em 20 de abril de 2020.

12. Disponível em: https://www.ppgihd-open-lab.com/post/nada-\%C3\%A9-t\%C3\%A3o-ruim-quen\%C3\%A3o-possa-piorar. Acesso em 20 de abril de 2020.

13. Algo semelhante também foi avaliado no estado de São Paulo. Consultar: https:// saude.estadao.com.br/fotos/geral,mapa-elaborado-pela-unesp-mostra-a-distribuicao-do-virusno-estado-de-sao-paulo-ao-longo-dos-eixos-rodoviarios,1088341. Acesso em: 17 de abril de 2020. 14. Consultar a contribuição de Fabio Rossi, "Volta Redonda: Números da pandemia de COVID-19”, disponível em: https://www.ppgihd-open-lab.com/post/volta-redondan\%C3\%BAmeros-da-pandemia-de-covid-19. Acesso em 17 de abril de 2020.

15. Em recente reflexão intitulada "'Nada é tão ruim que não possa piorar': A COVID-19 e as políticas de saúde na Baixada", André Santos da Rocha analisou a precariedade da região em termos de leitos hospitalares e vagas em UTI (Consultar: https://www.ppgihd-open-lab.com/ post/nada-\%C3\%A9-t\%C3\%A3o-ruim-que-n\%C3\%A3o-possa-piorar. Acesso em: 17 de abril de 2020). Da mesma maneira, uma nota técnica assinada por pesquisadores da ENSP-Fiocruz sobre "Limites e possibilidades dos municípios brasileiros para o enfrentamento dos casos graves de COVID19" lista apenas Duque de Caxias, Nilópolis e Itaguaí, dentre os treze municípios da Baixada Fluminense como parte do conjunto que possui o mínimo necessário de "estrutura hospitalar para o enfrentamento da covid-19". (Consultar: https://portal.fiocruz.br/sites/portal.fiocruz.br/ files/documentos/nota_tecnica_limites_e_possibilidades_municipios_covid-19.pdf. Acesso em: 17 de abril de 2020)

16. Consultar: https://www.economist.com/graphic-detail/2020/04/03/covid-19s-death-tollappears-higher-than-official-figures-suggest. Acesso em: 16 de abril de 2020.

17. Consultar: https://www.nytimes.com/2020/04/05/us/coronavirus-deaths-undercount.html. Acesso em: 16 de abril de 2020.

18. Consultar: https://oglobo.globo.com/sociedade/coronavirus/coronavirus-para-cientistasinfeccao-continuara-ser-uma-ameaca-global-por-ate-dois-anos-24355407. Acesso em: 16 de abril de 2020.

19. Consultar: https://noticias.uol.com.br/colunas/marcos-silveira/2020/04/06/covid-19-ografico-da-curva-esta-nos-enganando.htm?cmpid=copiaecola. Acesso em: 16 de abril de 2020.

20. Consultar: https://noticias.uol.com.br/ultimas-noticias/deutschewelle/2020/04/08/nao-enormal-dizem-coveiros-sobre-trabalho-em-cemiterios-de-sao-paulo.htm. Acesso em: 15 de abril de 2020.

21. Consultar: http://painel.covid19br.org/. Acesso em: 17 de abril de 2020

22. Consultar: https://www.abrasco.org.br/site/outras-noticias/saude-da-populacao/ estimativas-do-impacto-da-covid-19-na-mortalidade-no-brasil/46151/. Acesso em 17 de abril de 2020.

23. Consultar: https://noticias.uol.com.br/saude/ultimas-noticias/reuters/2020/04/08/ coronavirus-mata-mais-negros-em-estados-dos-eua-indicam-dados-preliminares.htm. Acesso em 17 de abril de 2020. Da mesma maneira, sugerimos a leitura de "Vida e morte na cidade: Segregação e exclusão na era da pandemia", de Mariana dos Santos Nesimi e Maurilio Lima 
Botelho, disponível em: https://www.ppgihd-open-lab.com/post/vida-e-morte-na-cidadesegrega\%C3\%A7\%C3\%A3o-e-exclus\%C3\%A3o-na-era-da-pandemia. Acesso em: 20 de abril de 2020. 24. Consultar: https://www.nytimes.com/2020/04/05/opinion/coronavirus-socialdistancing.html? action=click\&module=Opinion\&pgtype=Homepage\&fbclid=IwAR0w95WRvDjzs85dgwgn27l8Sjae1pZ91JzXg7d8JVsiDLtrkgxPjAumoqo. Acesso em 17 de abril de 2020.

25. Consultar: https://noticias.uol.com.br/reportagens-especiais/comunidade-no-rio-tem-50casos-de-tuberculose-e-poucas-defesas-contra-o-coronavirus/. Acesso em 17 de abril de 2020.

26. O prefeito Washington Reis, recentemente contaminado pelo coronavírus, foi o último alcaide da Baixada Fluminense a restringir a circulação de pessoas na cidade que administra, neste caso, Duque de Caxias. Em 14 de abril, a cidade já registrava 20 óbitos pela covid-19, apesar de ter registrado apenas 92 casos, uma assustadora taxa de letalidade de 21,7\%; para comparação, a taxa de letalidade da Itália, a maior do mundo, alcançou, no momento de pico, 12,5\%. Consultar: https://projetocolabora.com.br/ods1/covid-19-recorde-de-mortes-em-caxias-temor-na-baixadatoda/?utm_source=Colabora\&utm_campaign=1117c0432e-

EMAIL_CAMPAIGN_2020_04_16_02_05\&utm_medium=email\&utm_term=0_7b4d6ea50c-1117c0432e-417969293. Acesso em: 17 de abril de 2020.

27. Consultar: https://oglobo.globo.com/rio/2272-coronavirus-baixada-fluminense-registra-75de-aumento-em-casos-de-covid-19-24355078?

utm_source=Facebook\&utm_medium=Social\&utm_campaign=0\%20Globo. Acesso em: 17 de abril de 2020.

\section{RESUMOS}

Após atingir as áreas centrais e mais ricas da cidade do Rio de Janeiro, a pandemia de COVID-19/ coronavírus começa a ampliar suas fronteiras. No interior do estado e nas áreas mais pobres, a COVID-19 poderá ter impactos muito violentos. Com índices sociais alarmantes e precárias condições de saúde pública, o impacto da pandemia na Baixada Fluminense é preocupante e pode ser tornar um desastre social.

After reaching the central and wealthier areas of the city of Rio de Janeiro, the COVID-19 / coronavirus pandemic begins to expand its borders. In the interior of the state and in the poorest areas, COVID-19 could have very violent impacts. With alarming social indices and precarious public health conditions, the impact of the pandemic in the Baixada Fluminense is worrying and could be turning into a social disaster.

Después de llegar a las áreas centrales y más ricas de la ciudad de Río de Janeiro, la pandemia de COVID-19 / coronavirus comienza a expandir sus fronteras. En el interior del estado y en las áreas más pobres, COVID-19 puede tener impactos mucho más violentos. Con índices sociales alarmantes y precarias condiciones de salud pública, el impacto de la pandemia en la Baixada Fluminense es preocupante y podría convertirse en un desastre social.

Après atteindre les espaces centraux et plus riches de la ville de Rio de Janeiro, la pandémie covid-19 commence à élargir ses frontières. A l'intérieur de l'état de Rio de Janeiro et dans les espaces les plus pauvres, les impacts de la covid-19 pourront être très violents. A cause de la 
précarité de la santé publique et des indices sociaux alarmantes, les effets de la pandémie sur la Baixada Fluminense sont assez préoccupants et peuvent devenir un désastre social.

\section{ÍNDICE}

Keywords: COVID-19, coronavirus, Baixada Fluminense, metropolitan periphery, Rio de Janeiro. Palavras-chave: COVID-19, coronavírus, Baixada Fluminense, periferia metropolitana, Rio de Janeiro.

Palabras claves: COVID-19, coronavirus, Baixada Fluminense, periferia metropolitana, Río de Janeiro.

Mots-clés: COVID-19, coronavirus, Baixada Fluminense, périphérie métropolitaine, Rio de Janeiro.

\section{AUTORES}

\section{ALEXANDRE FORTES}

Professor Associado da Universidade Federal Rural do Rio de Janeiro na área de História Contemporânea, vinculado ao Departamento de História e Economia do Instituto Multidisciplinar, que chefiou no período 2006-2008. Bolsista de Produtividade do CNPq e Cientista do Nosso Estado (Faperj). Pró-Reitor de Pesquisa e Pós-Graduação da UFRRJ. Integrante da Red Latinoamericana de Historia Global. Membro permanente dos corpos docentes do Programa de Pós-Graduação em História, que coordenou no período 2009-2011, do Mestrado Profissional em Ensino de História (ProfHist), e do Programa de Pós-Graduação Interdisciplinar em Humanidades Digitais (PPGIHD-UFRRJ). Possui graduação em História pela Universidade do Vale do Rio dos Sinos, mestrado e doutorado em História pela Universidade Estadual de Campinas e pósdoutorado junto ao CeNedic (USP), ao PPGHIS (UFRJ) à Duke University com apoio da CAPES. Email: fortes.ufrrj@gmail.com.

\section{LEANDRO DIAS DE OLIVEIRA}

Professor Associado do Departamento de Geografia (DGG-IA) da Universidade Federal Rural do Rio de Janeiro na área de Geografia Econômica e da Indústria. Licenciado e Mestre em Geografia pela UERJ - Universidade do Estado do Rio de Janeiro, Doutor em Geografia pela UNICAMP Universidade Estadual de Campinas e Pós-doutor em Políticas Públicas e Formação Humana da Universidade do Estado do Rio de Janeiro (PPFH-UERJ). Professor dos quadros permanentes do Programa de Pós-Graduação em Geografia (PPGGEO-UFRRJ) e do Programa de Pós-Graduação Interdisciplinar em Humanidades Digitais (PPGIHD-UFRRJ). Líder do Grupo de Pesquisa Reestruturação Econômico-Espacial Contemporânea, do Laboratório de Geografia Econômica e Política (REEC-LAGEP). Membro da RELAEE - Rede Latino-Americana Espaço e Economia. E-mail: ldiasufrrj@gmail.com.

\section{GUSTAVO MOTA DE SOUSA}

Professor Adjunto IV do Departamento de Geografia (DGG-IA) da Universidade Federal Rural do Rio de Janeiro na área de Cartografia Temática e Digital. Licenciado e Bacharel em Geografia pela UFF - Universidade Federal Fluminense, Mestre e Doutor em Geografia pela UFRJ - Universidade Federal do Rio de Janeiro. Professor do quadro permanente do Programa de Pós-Graduação em Geografia (PPGGEO-UFRRJ). Editor Associado do Anuário do Instituto de Geociências da UFRJ. 
Membro do Laboratório integrado de Geografia Física Aplicada (LiGA/UFRRJ); Laboratório de Cartografia (GEOCART/UFRJ) e Laboratório de Dimensões Sociais Aplicadas a Atividade Física e ao Esporte (LABSAFE/UFRRJ).E-mail: gustavoms@ufrrj.br. 